PM0-064 CAN INTESTINAL FAILURE DUE TO FISTULA BE MANAGED SAFELY OUT WITH THE NATIONAL CENTRES?

doi:10.1136/gutjnl-2012-302514b.64

M Quinn,* S Falconer, R Mckee. Department of Colorectal Surgery, Glasgow Royal Infirmary, Glasgow, UK

Introduction Type II Intestinal Failure (IF) requires multidisciplinary management at significant cost. Up to $50 \%$ of these patients will progress to home parenteral nutrition (PN). The aim of this study was to assess the outcomes of patients with type II intestinal failure due to small bowel fistulae in a tertiary referral centre and compare these with published data.

Methods Patients were identified from a prospectively collected database (January 1998-December 2009). Data were analysed retrospectively. Data were split into groups by diagnosis: Acute pancreatitis, Upper Gastrointestinal, Colorectal, Hepatobiliary, Inflammatory Bowel Disease (IBD) and "other" (trauma, mesenteric ischaemic, failed PEG/PEJ, vascular, gynaecology). Data were analysed using SPSS V.17.1; Pearson $\chi^{2}$ test and ANOVA, $\mathrm{p}=0.05$ denoting significance.

Results 190 fistulas in 186 patients (median age 64 years (20-96), M:F 103:83) required PN. 75 (39.5\%) developed following emergency admission, 63 (32.2\%) following elective admission and 52 (27.4\%) were in patients transferred from other hospitals because of the fistula. $160(84.2 \%)$ fistulas developed following surgery. Patients undergoing major HPB procedures were statistically more likely to develop a post-op fistula $(\mathrm{p}=0.031) .113(59.5 \%)$ fistulas settled with conservative treatment. $34(18 \%)$ patients died before any surgery. Overall mortality was 21\% (39 of 186 patients). Patients with HPB pathology or those transferred from other hospitals were statistically more likely to die as a result of their fistula ( $p=0.007)$. Patients with IBD, colorectal pathology and those transferred from other hospitals were more likely to require surgical intervention for their fistula $(p=0.007)$. Patients in the "other" diagnosis category were statistically more likely to require a definitive operation $(p=0.02) .11$ fistulas required early open operation within 1-month. One due to underlying malignancy and ten to control sepsis. Following early operation one patient died, two required permanent home PN and five settled after open drainage of sepsis only. Two patients went on to further definitive surgery. 30 definitive operations were performed. Post operatively, four patients died, two required permanent HPN and three fistulas recurred in two patients, one of whom required further surgery.

Conclusion Reasonable outcomes from intestinal failure can be achieved out with the national referral services but a significant amount of resource is needed, including a multidisciplinary nutrition team, interventional radiology and a surgical team accustomed to dealing with such cases.

Competing interests None declared.

\section{PM0-065 REPAIR OF FRACTURED CENTRAL VENOUS CATHETERS FOR PARENTERAL NUTRITION: TECHNIQUES AND RISK OF INFECTION}

doi:10.1136/gutjnl-2012-302514b.65

M Small, ${ }^{*}$ G A D Major, S M Gabe. Lennard-Jones Intestinal Failure Unit, St Mark's Hospital, London, UK

Introduction Tunnelled central venous catheters (CVCs) are the best method of vascular access for long-term parenteral nutrition. Failure of intravenous access can necessitate emergeny admission. Removing and replacing CVCs is painful, risks infection and venous thrombosis, and uses costly healthcare resources. Simple techniques to repair fractures in the external part of CVCs can prolong CVC life but repairs have been associated with increased rates of catheterrelated bloodstream infection (CRBSI).
Methods Between January 2010 and December 2011 all repairs of CVC fractures were recorded. Fractures were defined as "external" when only the outer sheath of the CVC was ruptured, and "full" when both inner and outer sheaths of the CVC were ruptured, with evidence of leakage. External fractures were repaired with waterproof, non-absorbent tape. Full fractures were repaired with the manufacturer's repair kit, including a metal spike inserted into the lumen across the new join. We recorded rates of successful repair and any instance of CRBSI within 30 days of the repair.

\section{Results}

\begin{tabular}{llllll}
\hline Fracture type & $\begin{array}{l}\text { Repair } \\
\text { success (\%) }\end{array}$ & $\begin{array}{l}\text { Infection } \\
\text { rate (\%) }\end{array}$ & Detail & $\begin{array}{l}\text { Fracture rate/ } \\
\mathbf{1 0 0 0} \text { catheter-days }\end{array}$ \\
\hline Full & 34 & $30(88)$ & $1(3)$ & $\begin{array}{l}\text { Klebsiella } \\
\text { grown @ 30 days }\end{array}$ & 0.2 \\
External & 16 & $16(100)$ & 0 & - & 0.09 \\
Total & 50 & $49(98)$ & $1(2)$ & - & 0.3 \\
\hline
\end{tabular}

Conclusion CVC fracture repair is effective and safe. Repair of external fracture is easily done with waterproof, non-absorbable tape. Formal repair can prevent emergency admission and act a bridge to planned CVC replacement. It may also have a role in further prolonging the useful life of tunnelled CVCs. In our patient cohort repair is not associated with subsequent infection.

Competing interests None declared.

\section{REFERENCE}

1. Lundgren IS, Zhou C, Malone FR, et al. Central venous catheter repair is associated with an increased risk of bacteremia and central line associated bloodstream infection in pediatric patients. Pediatr Infect Dis J, 2011.

\section{PM0-066 SALVAGE OF OCCLUDED CENTRAL VENOUS CATHETERS IN LONG TERM PARENTERAL NUTRITION PATIENTS: TECHNIQUES \& OUTCOMES}

doi:10.1136/gutjnl-2012-302514b.66

G A D Major, M Small, ${ }^{*}$ S M Gabe. Lennard-Jones Intestinal Failure Unit, St Mark's Hospital, London, UK

Introduction Central venous catheter (CVC) occlusion is a recognised complication of parenteral nutrition (PN). Various techniques are advocated to salvage the CVC but forcing debris from fibrin or precipitate into the bloodstream risks causing catheter-related bloodstream infection (CRBSI) or embolism. We decided to assess the efficacy of simple physical techniques to salvage an occluded CVC, and the rate of complications described.

Methods All CVC occlusions between January 2010 and December 2011 were reviewed. Occlusions were "total", when the CVC could not be flushed, or "partial", when there was resistance to flushing. The cause of occlusion was recorded together with the time from symptom onset to attempted salvage, age of the CVC, techniques and instillations used to unblock the CVC, and outcomes. Confirmed cases of CRBSI occurring within 30 days of CVC salvage were recorded. Techniques included: manipulation of the external segment of the CVC to disrupt visible material $(\mathrm{M})$; saline flush of the CVC with a gentle, pulsing action using a $2.5 \mathrm{ml}$ Luer lock syringe for 20-60 min (SF); instillation of alcohol to dissolve lipid (A); pulsed flushing of urokinase without instillation (U); physical clearing out of the CVC hub with a 21G needle to remove any obstructing material (HC)

Results 38 occlusions occurred in 23 patients giving an occlusion rate of $10.9 \%$ (0.25 occlusions/1000 catheter days): 11 partial occlusions in five patients and 27 total occlusions in 19 patients. See Abstract PMO-066 table 1 below: The rate of CRBSI in this group 
was 0.02 CRBSI/1000 catheter days in the 2-year period assessed. This compares with a rate in patients not suffering occlusions of $0.91 \mathrm{CRBSI} / 1000$ catheter days.

\section{Abstract PM0-066 table 1}

\begin{tabular}{llll}
\hline Occlusion type & Detail & & Methods used \\
\hline Partial: $\mathrm{n}=11$ & Cause & 11 Lipid & $10 \mathrm{HC}$ \\
& Success rate & $10 / 11(91 \%)$ & $9 \mathrm{M}$ \\
& Failure & Used: M, SF, HC, A & $9 \mathrm{~S}$ \\
& & $2 \mathrm{~A}$ \\
Full: $\mathrm{n}=27$ & Cause & 9 Fibrin & $1 \mathrm{U}$ \\
& & 6 Lipid & $27 \mathrm{M}$ \\
& 1 Both & $25 \mathrm{SF}$ \\
& & 11 Unknown & $7 \mathrm{U}$ \\
& & $22 / 27$ (with lipid) \\
& Success rate & 2 Fibrin & \\
& Failures & 1 Lipid & \\
& & 1 Both & \\
& & 1 Unknown & \\
& & Used: $5 \mathrm{M}, 4 \mathrm{U}, 3 \mathrm{SF}, 2 \mathrm{U}, 1 \mathrm{~A}$ \\
& &
\end{tabular}

Conclusion CVC salvage is often possible with line manipulation and persistent pulsatile flushing. Hub clearout with a needle is also safe and effective. Alcohol and urokinase have an occasional role but are often not required and may not work without additional measures. Post-salvage complications are rare. The apparent negative association between occlusion and infection runs contrary to the belief that infection and occlusion are linked, and warrants further study.

Competing interests None declared.

\section{PM0-067 A RESPONSE TO NCEPOD (NATIONAL CLINICAL ENQUIRY INTO PATIENT OUTCOME AND DEATH 2010) REPORT-PRACTICE IN PROVISION OF PARENTERAL NUTRITION (PN) IN A REGIONAL REFERRAL CENTRE}

doi:10.1136/gutjnl-2012-302514b.67

${ }^{1} \mathrm{~N}$ Hamid, ${ }^{* 2} \mathrm{~A}$ Rochford, ${ }^{2} \mathrm{~L}$ Soo, ${ }^{2} \mathrm{~L}$ Barret, ${ }^{2} \mathrm{~N}$ Grasso, ${ }^{2} \mathrm{R}$ Ali, ${ }^{2} \mathrm{~F}$ Rahman, ${ }^{2} \mathrm{~A}$ Forbes. ${ }^{1}$ Department of Nutrition and Dietetics, University College London Hospitals, London, UK; ${ }^{2}$ GI Services, University College London Hospitals, London, UK

Introduction The externally peer-reviewed NCEPOD report into the provision of PN alarmingly identified suboptimal care in $81 \%$ of patients receiving $\mathrm{PN}$. We audited the quality of care received by parentally fed patients at a specialist regional centre for intestinal failure and home parenteral nutrition.

Methods Data were collected retrospectively for 100 inpatients seen between April and December 2011 on an NCEPOD modified audit tool; case notes were reviewed. Type I and II intestinal failure patients were included; home parenteral nutrition patients were excluded from the study.

Results Mean number of days patients were fed on PN was 12.2 (NCEPOD 13.2) and the range was $1-276$ days (NCEPOD 1-212). $10 \%$ of patients were fed for 3 days or less (NCEPOD 10\%) and $93 \%$ were fed for 30 days or less (NCEPOD 61\%). The top two specialities referring patients were Colorectal $27 \%$ and Oncology $18 \%$ as compared to $22 \%$ from general surgery and $20 \%$ started in ICU in NCEPOD report. $12 \%$ referrals were deemed inappropriate and not started PN (NCEPOD 15\%).

Conclusion Our hospital is a regional centre for intestinal failure and PN is prescribed exclusively through the nutrition support team (NST). Thus in comparison with the NCEPOD findings, the PN service at UCLH is of a high standard. Data collection were suffi- cient for direct comparison with most NCEPOD report parameters. The NST appeared to prevent inappropriate use of PN by not starting $12 \%$ of referred patients. Although a similar proportion of our patients $(10 \%)$ were fed for 3 days or less as found in the NCEPOD report, half of these patients were commenced on TPN in ICU. We found much lower rates of PN-related metabolic and catheter-related complications compared with the NCEPOD report because of active NST monitoring and good quality nursing care. Retrospective data collection on IV fluid prescribing was not possible, but it was perceived that there was some inappropriate use of IV fluids. A comprehensive database is being developed to enable prospective data collection to evaluate and develop the service further.

Abstract PM0-067 Table 1

\begin{tabular}{llr}
\hline Outcome & NCEPOD & UCLH \\
\hline Nutrition support team (NST) involvement & $52.7 \%$ & $100 \%$ \\
Appropriate first prescription & $85 \%$ & $100 \%$ \\
Adequate consideration of EN & $67 \%$ & $100 \%$ \\
Adequate biochemical/nutritional assessment prior & $46 \%$ & $100 \%$ \\
Documentation of nutritional assessment & $48 \%$ & $100 \%$ \\
Appropriate indication for PN & $71 \%$ & $95 \%$ \\
Adequate monitoring of PN patients & $56.7 \%$ & $100 \%$ \\
PN-related metabolic complications & $40 \%$ & $5 \%$ \\
Catheter-related complications & $26 \%$ & $5 \%$ \\
\hline
\end{tabular}

Competing interests None declared.

\section{REFERENCE}

1. NCEPOD. A Mixed Bag: The 2010 Report of the National Confidential Enquiry into Parenteral Nutrition. London: NCEPOD, 2010.

\section{PM0-068 TIMING OF PARENTERAL NUTRITION ADMINISTRATION SET CHANGES: IMPACT ON INCIDENCE OF CATHETER ASSOCIATED INFECTIONS}

doi:10.1136/gutjnl-2012-302514b.68

R Driver, ${ }^{*}$ P Mistry, D Swain, E Colinese, R Saich. Basingstoke and North Hampshire Hospital, Basingstoke, UK

Introduction Preparations of total parental nutrition (TPN) are stable for $48 \mathrm{~h}$. For patients with requirements $<2.51 / 24 \mathrm{~h}$ individual bags of TPN are delivered for more than $24 \mathrm{~h}$, resulting in cost savings and less frequent manipulations of the administration set. The epic2 guidelines recommend that administration sets exposed to lipids must be changed every $24 \mathrm{~h}$ to reduce possible line sepsis, effectively prohibiting the delivery of TPN bags over a period $>24 \mathrm{~h}$. The objective of this study is to determine whether the incidence of central venous catheter (CVC) associated infection is increased by running TPN over a period of $>24 \mathrm{~h}$ compared to $<24 \mathrm{~h}$.

Methods We analysed the CVC infection rate for 550 patients receiving TPN over a 3-year period, a total of 8339 line days. We assigned each CVC line into two groups; those through which at least one bag of TPN had been run for more than $24 \mathrm{~h}$ (1063 CVC lines) and those in which every bag was changed within $24 \mathrm{~h}$ (166 CVC lines). CVC-associated infection was confirmed when the same organism was grown on central and peripheral blood cultures, or blood culture and CVC tip culture. Information on CVC line position, duration and complication rate was also collected.

Results The CVC infection rate was 3.26/1000 line days in the $>24$ h group (95\% CI 3.0 to 3.5 ) and $3.14 / 1000$ line days in the $<24 \mathrm{~h}$ group (1.6 to 4.7 ). There was no significant difference in the infection rate between the two groups $\left(\chi^{2}, p=0.84\right)$. 